\title{
Electron Temperature Gradient Scale at Collisionless Shocks
}

\author{
Steven J. Schwartz, ${ }^{*}$ Edmund Henley, and Jeremy Mitchell \\ Blackett Laboratory, Imperial College London, South Kensington, London SWr 2AZ, UK \\ Vladimir Krasnoselskikh \\ Laboratoire de Physique et Chimie de l'Environnement et de l'Espace, CNRS, Orléans, France
}

(Dated: Physics Review Letters: Accepted 13 October 2011)

\begin{abstract}
Shock waves are ubiquitous in astrophysics and interplanetary space. In collisionless plasmas they transform directed flow energy into thermal energy and accelerate energetic particles. The energy repartition amongst particle populations is a multi-scale process related to the spatial and temporal structure of the electromagnetic fields within the shock layer. While major features of the large scale ion heating are known, the electron heating and smaller scale fields remain poorly understood and controversial. We determine for the first time the scale of the electron temperature gradient via unprecedented high time resolution electron distributions measured in situ by the Cluster spacecraft. We discover that half of the electron heating coincides with a narrow dispersive layer several electron inertial lengths $\left(c / \omega_{p e}\right)$ thick. Consequently, the nonlinear steepening is limited by wave dispersion. The DC electric field associated with the electron pressure gradient must also vary over these small scales, strongly influencing the efficiency of shocks as cosmic ray accelerators.
\end{abstract}

PACS numbers: 52.35.Tc,95.30.Qd,94.05.-a

Keywords: plasma shock waves, astrophysical plasmas, space plasmas

\section{BACKGROUND}

Shock waves abound throughout astrophysics, wherever fast flows encounter obstacles or other flows. Quantitative progress on the unequal energy partition at collisionless magnetised shock waves requires an understanding of the physics that occurs within the shock transition layer. To date, the primary experimental evidence for both shock acceleration and other dissipative processes has come from in situ measurements taken by instrumentation onboard spacecraft. Traversals of the bow shock formed by the impingement of the super-Alfvénic solar wind flow on the Earth's magnetosphere, and of interplanetary shocks, have been the richest source of knowledge (see, e.g. [1-3] and a recent comprehensive review [4]).

In theory, low Mach number shocks could dissipate the necessary energy entirely through some anomalous resistivity within the current-carrying shock layer. Moreover, right-hand fast magnetosonic/whistler waves have phase and group velocities that increase with decreasing wavelength beyond the fluid regime. Thus, steepened fast mode shocks may be expected to radiate short wavelength waves, and hence energy, into the unshocked oncoming flow. The shortest wavelength capable of standing in the flow then forms a "precursor wavetrain" that has been observed at these sub-critical shocks [5].

Above a critical Mach number, anomalous resistivity within the layer carrying the limited shock current is unable to convert the required amount of energy from directed bulk flow into thermal energy. At quasiperpendicular shocks, where the magnetic field in the unshocked region makes an angle $\theta_{B n}>45^{\circ}$ with the shock propagation direction (the shock "normal" $\hat{\boldsymbol{n}}$ ), a fraction of the incident ions are reflected by the steep shock ramp. They gyrate around the magnetic field and gain energy due to the transverse motional electric field $(-\boldsymbol{V} \times \boldsymbol{B})$. Their gyration returns them to the shock layer where they now have sufficient energy to pass through into the downstream shocked region [6]. This dispersal in velocity space represents an increase in peculiar velocity relative to the bulk motion and corresponds to the kinetic "heating" required by the shock jump conditions. The reflection occurs due to a combination of magnetic forces and an electrostatic cross-shock potential. The main potential, which corresponds to the frame-invariant $\boldsymbol{E} \cdot \boldsymbol{B}$ electric field, is known as the deHoffmann-Teller potential $[7,8]$. It results directly from the leading electron pressure gradient term in the Generalised Ohm's Law [9]. In more detailed two-fluid descriptions, the shock has fine structure that depends upon the characteristics of the nonlinear shock profile $[10,11]$.

By contrast, the electron heating problem has remained controversial. The action of DC shock fields on the electron population (which can have thermal speeds far in excess of the shock speed) is to inflate and open up a hole in the phase space distribution by accelerating (decelerating) incoming (escaping) electrons [12]. This inflation is not strictly dissipation or heating as it is reversible. Irreversibility is imposed by presumed scattering that infills the hole from the remnants of the nowaccelerated peak of the thermal electrons or by some other non-adiabatic process. Evidence for Debye-scale electric fields [13] suggests the phase space inflation is indeed accompanied by instabilities which could scatter the electrons. Demagnetisation of the electrons due to the strong gradient in the electric field [14] or nonlinear wave phenomena [15] offer alternative scattering processes. 
Thus the partition of energy between ions and electrons is a complex, self-consistent interplay between electron heating, magnetic field profile, shock potential, and ion reflection. This interplay remains poorly understood despite 40 years of research. That research has included detailed case studies [9], statistics of the inferred potential and electric field structures $[16,17]$, theoretical studies $[10,11]$ and increasingly sophisticated numerical simulations $[18,19]$.

Clues to the physics of the shock transition layer should be found by measuring its thickness. If the electron heating can be attributed to kinetic instabilities, the shock thickness will be measured in ion inertial lengths $\left(c / \omega_{p i}\right)$ $[20,21]$. If such instabilities prove ineffective, above a second critical Mach number the shock steepening is expected to be limited by whistler dispersion and/or be unstable to shock reformation [15]. Recent studies of the shock thickness [22] do show scales comparable to whistler wavelengths. These contrasted an earlier study [23] reporting scalings that matched the gyro-scales of reflected ions.

To date, studies have relied on the high temporal cadence available from magnetic or electric field experiments. However, field profiles provide only indirect evidence of the shock dissipation scales. A recent study [24] used sub-populations of electrons to determine the electrostatic potential profile at one shock, suggesting that it rose in concert with the magnetic field. In the present work, we resolve the electron population at sufficient cadence to reveal directly for the first time the scale of the electron temperature profile.

\section{METHOD}

The 4 Cluster spacecraft [25] are unique in their ability to remove the time-space ambiguity in time series data taken by in situ space plasma instrumentation. By timing the passage of an event at each corner of the tetrahedron formed by the 4 spacecraft, the planar orientation and speed of the event can be determined. We employ this technique to convert the time series of data to distance along the shock normal [26]. Fig. 1 illustrates the identification of the steep shock ramp that we use as event times.

The electron instrument on Cluster measures fluxes at several energies in a half-plane containing the spacecraft spin axis. These measurements form an azimuthal wedge divided into 12 polar directions from aligned to anti-aligned with the spin axis, and are repeated at 125$250 \mathrm{~ms}$ intervals. A full 3D distribution covering all azimuths is thus built up over 1 spin $(\sim 4 \mathrm{~s})$. However, when the magnetic field is roughly aligned with the spin axis, each wedge contains a full set of pitch angles from $0^{\circ}$ to $180^{\circ}$. Under these circumstances, and assuming gyrotropy, the full pitch angle distribution function is

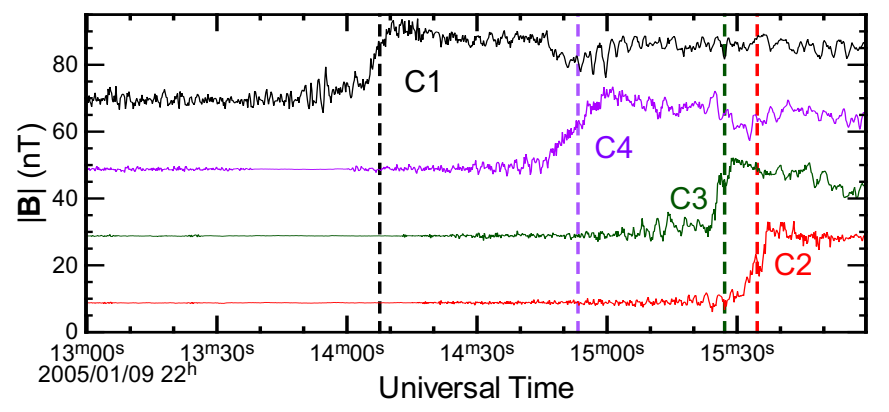

FIG. 1. (Color online) Magnetic field data at a crossing of the Earth's bow shock by the 4 Cluster spacecraft on 9 Jan. 2005. Traces have been shifted by $20 \mathrm{nT}$ for clarity. The dashed lines show the times of the steep ramp.

available at $\leq 250 \mathrm{~ms}$ resolution.

We rebin the raw electron data into pitch angles $\alpha$ relative to the instantaneous magnetic field. We calculate pseudo-densities and temperatures for each pitch angle bin as if the distribution were isotropic, e.g., $n\left(90^{\circ}\right)=$ $4 \pi \int f\left(v, \alpha=90^{\circ}\right) v^{2} d v$. These pseudo-moments better characterise the phase space distributions in the $\|, \perp$ directions than the full $T_{\|, \perp}$ moments [cf. Fig 9 of 27].

\section{RESULTS AND CONCLUSIONS}

An overview of the data for 2005 Jan 9 is shown in Fig. 2. The transition from unshocked solar wind plasma to the shocked magnetosheath occurs around 22:15:30. Although the solar wind flow is a factor of 10 slower than the electron thermal speed, some residual modulation at the spin period is evident in the data. We have averaged the parallel and anti-parallel $\left(\alpha=0,180^{\circ}\right)$ moments so that the second and third panels of Fig. 2 reveal the pseudo-parallel and perpendicular moments. Note that the pseudo-densities $n(\alpha)$ are not, and from their definition above need not be, equal. The bottom two panels show increasing oscillations and a gradual "foot" ahead of a steeper magnetic "ramp" region. The dominant $\hat{z}$ magnetic field component is nearly aligned with the spin axis, enabling the parallel and perpendicular moments to be available in every $0.25 \mathrm{~s}$ wedge as described above. Fig. 2 already suggests our main result: the rise in electron temperature follows closely even the steepest ramp of the magnetic field.

Fig. 3 shows that both the parallel and perpendicular electron temperatures closely track the steep rise in magnetic field, with half the electron heating taking place on a scale of $17.3 \mathrm{~km}$, corresponding to 6.4 electron inertial lengths and a small fraction (0.15) of an ion inertial length. Although much of the electron dynamics is linked to the DC electric and magnetic fields within the ramp $[8,24,28,29]$ and is therefore reversible, the fact that both $T_{e \|}$ and $T_{e \perp}$ rise together suggests an infla- 


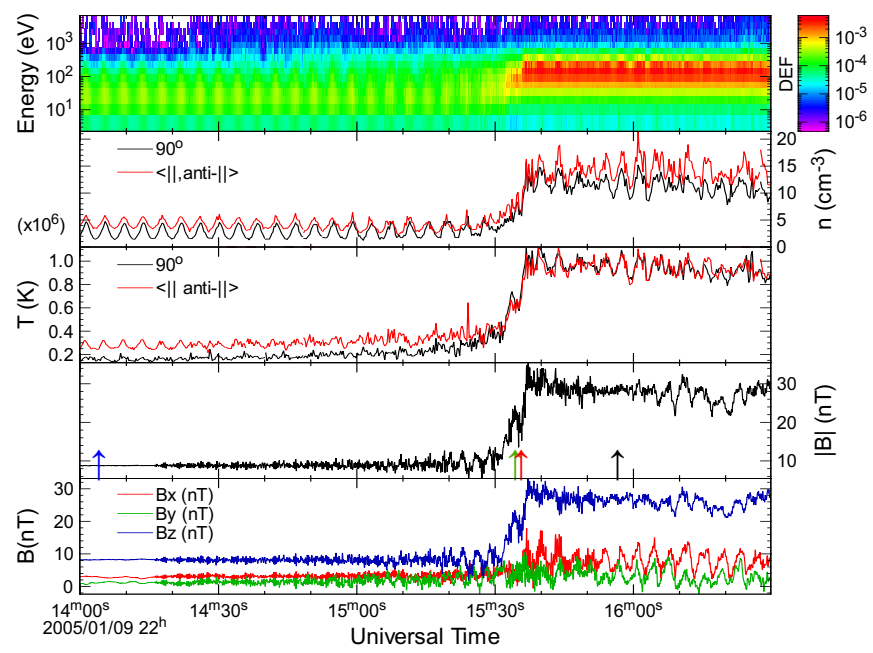

FIG. 2. (Color online) Overview of data from Cluster 2 on 2005 Jan 9. From top to bottom: Omni-directional electron energy-time spectrogram @ $250 \mathrm{~ms}$ resolution, electron pseudo-density, electron pseudo-temperatures (see Method), magnetic field magnitude, and field components. Arrows in the fourth panel show locations of the cuts presented in Fig. 4.

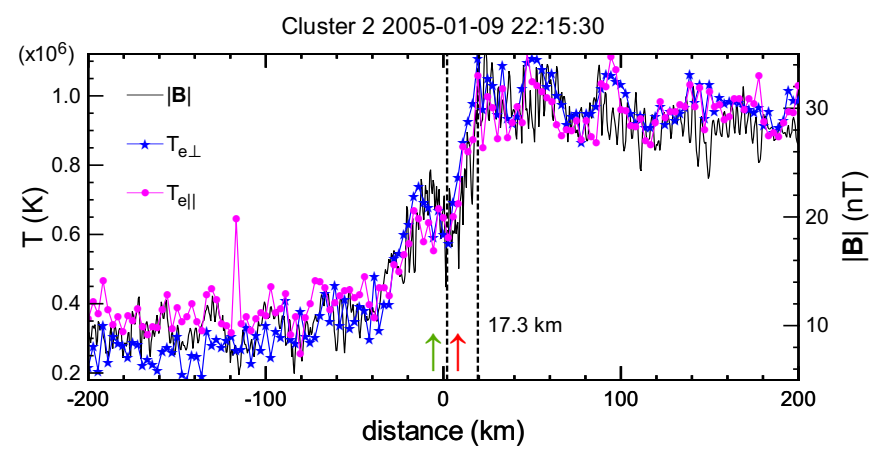

FIG. 3. (Color online) Magnetic field (solid) and electron temperature (symbols) as a function of distance from the shock ramp. Roughly half the temperature rise occurs within the region $17.3 \mathrm{~km}$ wide between the dashed vertical lines corresponding to 6.4 electron inertial lengths $\left(c / \omega_{p e}\right)$.

tion of the particle phase space distribution that is not reversible, due primarily to the filling in and/or entrapment of electrons in regions of phase space that would otherwise be inaccessible.

This infilling can be seen in the cuts of the distributions shown in Fig. 4. Within the steep ramp, the inflated distribution is evident, with the flat-topped infilled region already at its downstream level. This supports the notion that the temperature profiles shown in Fig. 3 really do represent irreversible heating. Interestingly, Fig. 4 shows that features previously reported with the ramp, e.g., the beam vestige of the solar wind peak [28], are present only in the more gradual initial rise that precedes the steep ramp. That beam has been totally eroded by the time this electron scale ramp is encountered.

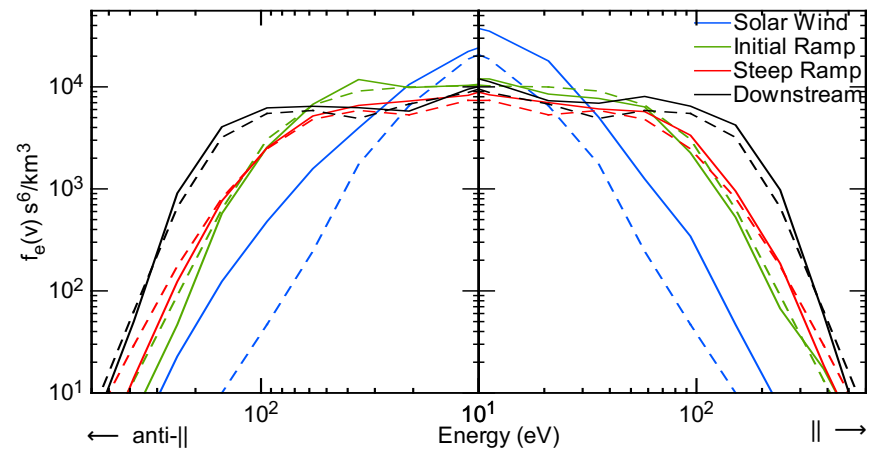

FIG. 4. (Color online) Cuts of the electron distribution functions in the solar wind, initial ramp, steep ramp, and downstream along (solid) and perpendicular (dashed) to the magnetic field. The locations of the cuts are indicated along the axes in Figs. 2 and 3. Note the solar wind halo drift evident in the anti-aligned direction and the absence of features within the steep ramp.

Thus the electron heating occurs over scales that are significantly smaller than the convected proton gyro-scale $V_{n} / \Omega_{c i s}$ invoked in [23] and also smaller than the ion inertial length that might be anticipated due to microinstabilities within the shock current layer [20,21].

Recent statistical studies [22] argued that previous fits to a proxy of the plasma density profile [23] mixed contributions from the more extended foot region governed by reflected gyrating ions. Restricting the measurements to just the steep ramp, they report widths in the range $3-55 c / \omega_{p e}$ with a decreasing trend as the Mach number increases. They interpreted their work in terms of shock steepening limited by the dispersion of electron whistler waves, with dispersion relation $\omega=$ $\Omega_{e} \cos \theta_{B n}\left(k^{2} c^{2} / \omega_{p e}^{2}\right)$. The limiting case of a wave capable of phase standing in the incident flow has a wavelength that can be written

$$
\frac{\lambda}{c / \omega_{p e}}=2 \pi \frac{\cos \theta_{B n}}{M_{A}} \sqrt{\frac{m_{p}}{m_{e}}}
$$

The results from Table I yield a value of 9.2 for this ratio, comparable to the 6.4 electron inertial lengths given above. The fact that supercritical shocks steepen to this whistler limit suggests that dissipation processes are insufficient to broaden the transition further.

It should come as no surprise that the steepening of a fast mode (right-handed) wave results in a right-handed whistler signature. Indeed, the non-coplanar component of the magnetic field [30], responsible for the difference in the shock electrostatic potential when viewed in different shock rest frames [8], is right-handed. There is new evidence [31] that the wave Poynting flux is directed away from the ramp region upstream as expected for dispersion-limited steepening.

The present study measures directly the actual temperature profile of the electrons. The result confirms that 
TABLE I. Shock Parameters 2005 Jan 9 @ 22:15

\begin{tabular}{lr}
\hline Parameter & Value \\
\hline$V_{\text {shock }}$ & $+10.8 \mathrm{~km} / \mathrm{s}$ \\
Unshocked magnetic field $\boldsymbol{B}_{u}{ }^{\dagger}$ & $(3.07,1.35,8.14) \mathrm{nT}$ \\
Unshocked electron density & $4.0 \mathrm{~cm}^{-3}$ \\
Location (Earth radii) & $(12.3,13.3,-6.7) R_{e}$ \\
$\hat{\boldsymbol{n}}$ shock normal (timing) & $(0.855,0.418,-0.307)$ \\
$\hat{\boldsymbol{n}}$ (model) $[26]$ & $(0.904,0.383,-0.189)$ \\
$V_{n} \equiv \boldsymbol{V} \cdot \hat{\boldsymbol{n}}$ (shock rest frame) & $373 \mathrm{~km} / \mathrm{s}$ \\
Alfvén Mach no. $M_{A}$ & 3.8 \\
Magnetosonic Mach no. $M_{m s}$ & 3.0 \\
$\theta_{B n u} \equiv \angle \boldsymbol{B}, \hat{\boldsymbol{n}}$ & 83 \\
Plasma ion $\beta_{i}$ & 0.4 \\
Plasma electron $\beta_{e}$ & 0.34 \\
Electron inertial length $c / \omega_{p e}$ & $2.7 \mathrm{~km}$ \\
Ion inertial length $c / \omega_{p i}$ & $117 \mathrm{~km}$ \\
$V_{n} / \Omega_{\text {ciu }} \dagger$ & $443 \mathrm{~km}$ \\
$V_{n} / \Omega_{c i s}$ & $139 \mathrm{~km}$ \\
Whistler wavelength $\lambda$ & $24.8 \mathrm{~km}$ \\
Electron larmor radius $r_{L e} u$ & $1.01 \mathrm{~km}$ \\
\hline$\dagger$ & All vectors are in the GSE frame of reference. Subscripts \\
" $s$ " (" $u$ ") denote quantities in the (un)shocked region. \\
$\dagger \dagger \Omega_{c i} \equiv e B / m_{p}$ is the proton gyrofrequency
\end{tabular}

nonlinear steepening proceeds down to scales limited by whistler dispersion. We have argued that this represents irreversible heating, implying that dissipation is operative on this, or probably smaller, scales.

We have attempted a similar analysis on other shock crossings observed by Cluster, with consistent findings. Suitable events are rare, since they require the combination of a slowly moving shock and favorable magnetic field orientations. Future space missions target electron physics and should provide numerous examples for statistical studies.

What process(es) are actually responsible for (sub-)whistler-scale dissipation? The overall inflation in phase space is linked to the action of the cross-shock electrostatic potential in concert with the magnetic mirror forces. Some or all of the potential may be concentrated in intense spikes [32] that may break the adiabaticity of electron phase space trajectories despite a ramp thickness which, in our example, is 20 times the local electron gyroradius. Candidate processes [e.g. 14] responsible for in-filling regions of phase space, in some of which electrons are trapped, include wave scattering $[12,33]$ and demagnetization [14]; these will require further analysis and simulations.

Our discovery of short scale electron heating has an important consequence for electron and ion acceleration. Gradient drift and surfing mechanisms are sensitive to the scale of the field transitions [34], becoming very efficient at scales comparable to those reported here.

This work was supported by the UK STFC and by a CNES "Cluster Co-I DWP" grant. We are grateful for the support of ISSI, Bern. Data were drawn from the Cluster Active Archive and/or instrument teams led by
A. Fazakerley, I. Dandouras, and E. Lucek. We thank the referees for helpful comments.

s.schwartz@imperial.ac.uk

[1] R. G. Stone and B. T. Tsurutani, Washington DC American Geophysical Union Geophysical Monograph Series 34 (1985).

[2] B. T. Tsurutani and R. G. Stone, Washington DC American Geophysical Union Geophysical Monograph Series 35 (1985).

[3] G. Paschmann, S. J. Schwartz, C. P. Escoubet, and S. Haaland, "Outer magnetospheric boundaries: Cluster results," in Space Science Reviews, Vol. 118 (2005).

[4] R. A. Treumann, A\&A Rev. 17, 409 (2009).

[5] M. H. Farris, C. T. Russell, and M. F. Thomsen, J. Geophys. Res. 98, 15285 (1993).

[6] N. Sckopke, G. Paschmann, S. J. Bame, J. T. Gosling, and C. T. Russell, J. Geophys. Res. 88, 6121 (1983).

[7] F. de Hoffmann and E. Teller, Physical Review 80, 692 (1950).

[8] C. C. Goodrich and J. D. Scudder, J. Geophys. Res. 89, 6654 (1984).

[9] J. D. Scudder, T. L. Aggson, A. Mangeney, C. Lacombe, and C. C. Harvey, J. Geophys. Res. 91, 11053 (1986).

[10] A. A. Galeev, V. V. Krasnosel'skikh, and V. V. Lobzin, Soviet Journal of Plasma Physics 14, 1192 (1988).

[11] M. Gedalin, Geophys. Res. Lett. 24, 2511 (1997).

[12] J. D. Scudder, A. Mangeney, C. Lacombe, C. C. Harvey, and C. S. Wu, J. Geophys. Res. 91, 11075 (1986).

[13] S. D. Bale, P. J. Kellogg, D. E. Larson, R. P. Lin, K. Goetz, and R. P. Lepping, Geophys. Res. Lett. 25, 2929 (1998).

[14] M. Balikhin and M. Gedalin, Geophys. Res. Lett. 21, 841 (1994).

[15] V. V. Krasnoselskikh, B. Lembège, P. Savoini, and V. V. Lobzin, Physics of Plasmas 9, 1192 (2002).

[16] S. J. Schwartz, M. F. Thomsen, S. J. Bame, and J. Stansberry, J. Geophys. Res. 93, 12923 (1988).

[17] S. Walker, H. Alleyne, M. Balikhin, M. André, and T. Horbury, Annales Geophysicae 22, 2291 (2004).

[18] B. Lembege, J. Giacalone, M. Scholer, T. Hada, M. Hoshino, V. Krasnoselskikh, H. Kucharek, P. Savoini, and T. Terasawa, Space Sci. Rev. 110, 161 (2004).

[19] M. Scholer and D. Burgess, Physics of Plasmas 13, 062101 (2006).

[20] K. Papadopoulos, Washington DC American Geophysical Union Geophysical Monograph Series 34, 59 (1985).

[21] S. Matsukiyo and M. Scholer, J. Geophys. Res. 111, A06104 (2006).

[22] Y. Hobara, M. Balikhin, V. Krasnoselskikh, M. Gedalin, and H. Yamagishi, J. Geophys. Res. 115, A11106 (2010).

[23] S. D. Bale, F. S. Mozer, and T. S. Horbury, Physical Review Letters 91, 265004 (2003).

[24] B. Lefebvre, S. J. Schwartz, A. N. Fazakerley, and P. Décréau, J. Geophys. Res. 112, A09212 (2007).

[25] C. P. Escoubet, C. T. Russell, and R. Schmidt, The Cluster and PHOENIX missions (Dordrecth: Kluwer, 1997).

[26] S. J. Schwartz, ISSI Scientific Reports Series 1, 249 (1998).

[27] J. Mitchell, S. J. Schwartz, and U. Auster, Annales Geo- 
physicae in press (2011).

[28] W. C. Feldman, R. C. Anderson, S. J. Bame, S. P. Gary, J. T. Gosling, D. J. McComas, M. F. Thomsen, G. Paschmann, and M. M. Hoppe, J. Geophys. Res. 88, 96 (1983).

[29] J. D. Scudder, Adv. Space Res. 15, 181 (1995).

[30] M. F. Thomsen, J. T. Gosling, S. J. Bame, K. B. Quest, and D. Winske, J. Geophys. Res. 92, 2305 (1987).
[31] D. Sundkvist, V. Krasnoselskikh, S. D. Bale, S. J. Schwartz, J. Soucek, and F. Mozer, Physical Review Letters under revision (2011).

[32] S. D. Bale and F. S. Mozer, Physical Review Letters 98, 205001 (2007), arXiv:physics/0703101.

[33] P. Veltri and G. Zimbardo, J. Geophys. Res. 981, 13335 (1993).

[34] G. P. Zank, H. L. Pauls, I. H. Cairns, and G. M. Webb, J. Geophys. Res. 101, 457 (1996). 